\title{
Author Index to Abstracts
}

Numbers refer to abstract numbers

Aguercif, M. 70

Akerblom, H. K. 48

Anagnou, E. 18

Anast, C. S. 60

Angelini, C. 67, 68

Angus, R. 17

Antsaklis, A. 39

Aronsson, M. 8

Aula, P. 87

Avery, A. 76

Aynsley-Green, A. 7, 45

Bachmann, C. 71

Ballowitz, L. 65

Barber, A. 36

Bartels, H. 29

Bastakis, N. 47

Basuyau, J. P. 25, 66

Battistella, P. A. 67,68

Baum, J. D. 17

Baumann, W. 12

Becker, M. A. 20

Bektas, S. 50

Beratis, N. G. 52

Betend, B. 4

Bethenod, M. 34

Betke, K. 2, 62

Bienvenu, F. 34

Bienvenu, J. 34

Bloom, S. R. 45

Blum, D. 77

Boller, E. 51

Bommer, J. 30

Bossi, E. 22

Bottini, E. 72, 73, 74

Bouhass, R. 70

Bourre, J. M. 84

Bower, B. D. 7

Boyes, S. 7

Brandeis, W. E. 80

Bremer, H. J. 35

Brendlein, F. 80

Brunelle, Ph. 66

Bucci, G. 59, 61

Bury, A. J. 36

Calame, A. 49

Carapella, E. 72, 73, 74

Carse, E. 78

Casaer, P. 10

Cassimos, Chr. 89

Cetre, E. 49

Chrzanowski, R. 49

Claridge, R. 46

Colarizi, P. 61

Colombo, J. P. 71

Constantopoulos, A. 58

Constantopoulos, C. 9

Corbeel, L. 1, 10

Dafforn-Ierodiaconou, E. 26

D'arcangelo, C. 91
David, L. 27, 32, 60

DeBoeck, K. 1, 10

DeLaunay, J. 70

Del Principe, D. 91

Delpy, D. 75

de Menibus, C. H. 66

Demopoulos, K. 31

Deonna, Th. 49

Desjeux, J. F. 21

Digeorge, A. M. 53

Discepoli, L. 73

Doménech, E. 33

Donzelli, F. 67, 68

Dubois, P. M. 60

Durand, P. 83

Duc, G. 51

Eaton, J. 7

Eeckels, R. 1, 10

Eggermont, E. 10

Eife, R. 2

Enders, A. 2

Eriksson, B. O. 15

Essich, H. J. 19

Fahrig, A. 23

Favart, A. M. 13

Ferro, R. 59, 61

Fessas, F. 9, 39

Forrest, G. C. 46

Francois, R. 4, 32, 86

Frederich, A. 27

Friberg, L. G. 15

Frobert, Y. 86

Fuhrmann, G. 62

Galanos, C. 62

Garabedian, M. 25

Garibaldi, L. R. 53, 83

Gautier, E. 84

Geubelle, F. 5

Gillet, P. 86

Gilli, G. 23

Gitzelmann, R. 54

Gloria-Bottini, F. 72, 74

Godet, J. 50

Gorodischer, R. 38

Gudinchet, F. 44

Guibaud, P. 27

Gyurkovits, K. 92

Hadjigeorgiou, E. M. 18, 69

Hahn, T. 14

Halsall, D. 75

Halvorsen, S. 11

Hammarlund, K. 43

Hanefeld, F. 56, 82

Heinz, F. 3

Helge, H. 88

Henderson-Smart, D. 78

Hermier, M. 4
Herschkowitz, N. 22

Hoting, E. 40

Hrbek, A. 41

Isacchi, G. 59, 61

Iversen, K. 41

Jäger-Roman-E. 88

Jequier, E. 44

Johnson, P. 78

Kafatos, A. G. 47

Kahn, A. 77

Karaiskos, K. 18

Karaklis, A. 37

Karayanopoulos, C. 39

Kaskarelis, D. 39, 69

Kasperek, K. 35

Kelson, S. 62

Kinmonth, A. L. 17

Kipourou, L. 31

Klose, H. J. 62

Knudtzon, J. 16

Koliouskas, D. 89

Kontopoulos, E. 89

Kraemer, R. 5, 6

Krempien, B. 30

Kreth, W. 3

Kreusser, W. 28

Kyllerman, M. 8

Lahet, C. 34

Lamy, M. E. 13

Lapatsanis, P. 26, 31, 37

Laszlō, A. 92

Laufer, D. 24

Laurenti, F. 59, 61

Lazarides, P. 69

Lennert, Th. 82

Lentze, M. J. 90

Leroyer, E. 60

Levin, S. 14

Levy, J. 13

Linderkamp, $0.62,79$

Lipp, A. 49

Lippmann, A. 63

Logghe, N. 10

Lolis, D. 69

Lombeck, I. 35

Loucopoulos, D. 39

Lucarelli, P. 72, 74

Lucarini, N. 72, 74

Lucas, A. 45

Lundblad, A. 87

Lutz, P. 19

Macri, F. 73

Malagnino, F. 61

Malaka-Zafiriu, K. 89

Malamitsi-Puchner, A. 69

Mallet, E. 25, 66

Mancuso, G. 91 
Mann, L. 36

Mansour, A. B. 21

Marget, W. 62

Marzetti, G. 59

Matsaniotis, N. 9, 58

Matteucci, P. 72

Matthieu, J. M. 84

Mehls, O. 23, 28

Mellander, M. 42

Mendicini, M. 61

Menichelli, A. 91

Mengreli, C. 26, 37

Michalk, D. 19

Micheli, J. L. 44, 49

Mordasini, R. 6

Morle, F. 70

Morle, L. 70

Morphis, L. 58

Moya, M. 33

Muller, W. 3, 63, 81

Muller-Wiefel, D. E. 80

Nakou, S. 37

Nicolopoulos, D. 18, 69

Niklasson, A. 42

Olegard, R. 8, 42

Olsson, T. 41

Ørstavik, I. 11

Orzalesi, M. 72

Otnaess, A. B. 11

Palmer, S. R. 76

Panagiotakopoulos, G. 47

Panero, A. 59

Pantelakis, S. 26, 47

Parker, D. 75

Pascone, R. 72

Paunier, L. 24

Pavlatou, M. 18

Peltonen, R. 64

Peltonen, T. 64

Perheentupa, J. 85

Persiani, M. 91
Peter, H. H. 3

Philippe, N. 86

Polidoro, E. 67, 68

Pollitzer, M. J. 75

Raivio, K. O. 20, 87

Rajantie, J. 85

Rapola, J. 57

Raptis, S. 18

Rating, D. 88

Reigner, J. 84

Renaud, H. 32

Renlund, M. 87

Reynolds, E. O. R. 75

Rezvani, I. 53

Rieger, C. H. L. 3, 63, 81

Riegel, K. P. 62, 79

Ritz, E. 23, 28, 30

Robinson, M. J. 76

Ronchetti, R. 73, 74

Rossi, E. 6

Rossini, M. 61

Rousson, R. 27

Rubaltelli, F. F. 67, 68

Sabel, K. G. 8, 15, 42

Salle, B. 32

Sandin, B. 8

Sann, L. 27, 34

Savignoni, P. G. 59

Sbyrakis, S. 31

Schachten, J. 30

Schärer, K. 80

Schneider, J. 90

Schoech, G. 40

Schoeni, M. 6

Schutz, Y. 44

Schwartz, M. 53

Schwartz, R. H. 7

Sedin, G. 43

Shaw, J. C. L. 36

Shinwell, E. 38

Siegert, M. 56
Siegrist, H. P. 22

Siemes, H. 56

Simell, O. 57, 85

Sipila, I. 57

Sizonenko, P. C. 24

Stallinger, H. 79

Steinmann, B. 54

Stettler, E. 44

Stokidou, M. 47

Stoltenburg, G. 82

Szabō, L. 92

Taylor, R. W. 76

Touraine, J. L. 4

Trakas, D. 47

Tramutoli, G. 73

Tuchschmid, P. 51

Vainsel, M. 13

Valassi-Adam, H. 9

Van den Berghe, G. I

Vandepitte, J. 10

Vannas, A. 57

Varenne, P. 32

Verbist, L. 10

Vergani, L. 67, 68

Vis, H. L. 77

Vretos, C. 31

Weinkauf, R. 28

West, R. J. 55

Whitehead, M. D. 75

Whyte, P. 78

Wiese, G. 65

Wiesmann, V. 22

Wilbur, L. 52

Wilkinson, A. 78

Wynder, E. 47

Zervas, J. 9

Zissis, G. 13

Zoumboulakis, D. 9

Zoupas, E. 18

Zuppinger, K. 22 